\title{
RECURSOS Y ESTRATEGIAS DE AFRONTAMIENTO AL ESTRÉS EN DOCENTES DE CENTROS ESCOLARES ESTATALES DE LIMA METROPOLITANA ${ }^{1}$
}

\section{Mario Bulnes Bedón}

Se estudia la relación entre los Recursos de afrontamiento y las estrategias de afrontamiento al estrés en 546 docentes de centros educativos estatales de Lima Metropolitana de los tres niveles de enseñanza: Inicial, Primaria y Secundaria. El diseño utilizado es el descriptivo comparativo y correlacional y los instrumentos aplicados son: El Inventario de Recursos de Afrontamiento de Hammer y Marting, y el Cuestionario de Modos de afrontamiento al estrés elaborado por Carver y adaptado por Casuso en 1996. Los resultados nos demuestran que existe un mayor predominio de los recursos cognitivos en todos los docentes evaluados. En cuanto a las estrategias de afrontamiento, los docentes de Inicial y Primaria prefieren utilizar como la planificación, reinterpretación positiva y crecimiento y acudir a la religión; mientras que los de Secundaria prefieren utilizar las estrategias de afrontamiento activo, planificación, reinterpretación positiva y crecimiento. Las comparaciones nos permiten observar que entre los docentes de Inicial y Primaria no existe diferencia estadísticamente significativa en los recursos de afrontamiento ni en cuanto a las estrategias de afrontamiento al estrés, pero sí se observan diferencias entre los docentes de Inicial y Secundaria, y entre los docentes de Primaria y Secundaria. Asimismo se observa correlación entre la variable recursos personales y la variable estrategias de afrontamiento al estrés.

PALABRAS CLAVE: Recursos de afrontamiento, estrategias de afrontamiento, estrés, docentes, salud.

The relation between the Resources of facing and the strategies of facing to stress in 546 educational ones of state educative centers of Lima Metropolitan of the three levels of education studies: Initial, Primary and Secondary. The used design is descriptive the comparative one and corelational and the applied instruments are: The Inventory of Resources of facing of Harnmer and Marting, and the Questionnaire of Ways of facing to the stress elaborated by Carver and adapted by Casuso in 1996. The results demonstrate to us that a greater predominance of the evaluated cognitivos resources in all the educational ones exists. As far as the facing strategies, the educational ones of Primary Initial and prefer to use like the planning, positive reinterpretacion and growth and to go to the religion; whereas those of Secondary prefer to use the strategies of active facing, planning, positive reinterpretación and growth. The comparisons allow us to observe that between the educational ones of Primary and Initial and statistically significant difference in the resources of facing does not exist nor as far as the strategies of facing to stress, but differences between the educational ones of Secondary Initial and, and between the educational ones of Primary and Secondary are observed. Also correlation between the variable is observed personal resources and the variable strategies of facing to stress.

KEY WORDS: Resources of facing, strategies of facing, stress, educational, health.

\footnotetext{
${ }^{1}$ Participaron en la investigación: Roger Elizalde, Renato Santiváñez, Rosa Huerta (Miembros A y E), Leni Alvarez. Eliana Delgado (Colaboradores)
} 


\section{INTRODUCCION}

Una de las funciones más importantes que cumplen los profesionales de la salud y especialmente el psicólogo, es la de brindar ayuda a las personas a que logren expresar una vida plena, desplegando todos sus recursos personales en la búsqueda de bienestar, lo que los humanistas llaman realización o también expresión de sus potencialidades. Ante esto, surge el concepto de estrés, el cual es un fenómeno psicológico de la vida moderna que permite comprender los actuales momentos que se vive en el país y que constituye serios obstáculos para conseguir el bienestar personal.

En este sentido, los procesos de ajuste ante los retos que la vida demanda a la población provocan variadas consecuencias, las cuales pueden ser positivas en algunos casos, como el logro de metas ante situaciones apremiantes, pero también pueden ser negativas, cuando el afrontamiento es inadecuado, como por ejemplo, disfunción familiar, violencia familiar, intolerancia, problemas psicosomáticos, problemas sociales, entre otros. Estas consecuencias influyen directamente en personas de diferentes edades, tanto en niños, adolescentes, jóvenes como en adultos y adultos mayores, sin importar los niveles socioeconómicos ni ocupacionales. Es así como el docente de entidades públicas no es ajeno a estas circunstancias, lo que podría entorpecer su labor como mediador del aprendizaje de sus alumnos y de la formación de futuros ciudadanos, quienes constituyen el recurso humano con el que cuenta el país para construir un futuro mejor.

Como ya se mencionó, el estrés psicológico es uno de los fenómenos más extendidos en los actuales momentos y afecta a las personas de diversas condiciones socioeconómicas y laborales. Se entiende que el estrés es fruto de la interacción entre una persona y su entorno, el cual aparece en la medida que exista una discrepancia entre las demandas que experimenta una persona y la capacidad de ésta para responder a ella, y al trastocar su crecimiento personal y su realización se convierte en una fuente de dolor y sufrimiento.

El concepto de estrés aquí planteado, se encuentra asociado a los conceptos de evaluación cognitiva -como un aspecto importante para evaluar el entorno del sujeto- y el de afrontamiento al estrés como proceso dinámico que responde a demandas objetivas y a evaluaciones subjetivas de la situación. Estos conceptos fueron planteados por Lazarus (1966) quien, además, refiere que «las personas y los grupos difieren en su sensibilidad y vulnerabilidad a ciertos tipos de acontecimientos, así como en su interpretación y reacción ante lo mismo». Es decir, las acciones que realiza una persona para afrontar un problema afecta la valoración del problema mismo y el consecuente afrontamiento.

En este sentido, la presente investigación considera como variables, por un lado, a las estrategias de afrontamiento ante las situaciones de estrés, las cuales pueden ser entendidas como «los esfuerzos cognitivos y conductuales orientados a manejar (reducir, minimizar, dominar o tolerar) las demandas internas y externas de una determinada situación estresante» (Lazarus y Folkman, 1986), y por otro lado, los recursos de afrontamiento al estrés, los que constituyen «características personales que conforman su capacidad adaptativa, la cual provee de inmunidad contra posibles daños producto de un evento estresante. Es un potencial para la acción pero no una acción en si misma» (Lazarus y Folkman, 1986).

Los recursos son considerados moderadores del estrés porque influyen en el momento de la evaluación de la experiencia como estresante y en la elección de determinadas estrategias (Taylor, 1991 y Thoits, 1995). Los recursos de afrontamiento pueden influir en la intensidad de las respuestas, en la interpretación del evento, en el esfuerzo puesto durante el afrontamiento o pueden incrementar los recursos personales para manejar de manera eficiente las situaciones estresantes (Zeidner y Hammer, 1992).

Se ha demostrado que las personas que poseen una gran variedad de recursos de 
afrontamiento ante situaciones estresantes experimentan pocas dificultades emocionales (Thoits, 1995), hasta cierto punto cumple una función protectora en cuanto:

1. Eliminan o modifican las condiciones que facilitan el desarrollo de problemas.

2. Controlan el significado de la experiencia, de manera que se neutraliza la situación problemática.

3. Mantienen las consecuencias emocionales dentro de los límites razonables.

Si bien es cierto que las situaciones de estrés antes descritas son comunes a todas las personas, en el caso de los docentes de centros educativos estatales toman una especial importancia toda vez que su rol principal como educadores. se encuentra asociado a la formación integral de niños y adolescentes y, por tanto, el contexto en el que se desenvuelven no sólo influirá en su desarrollo personal, sino también en el de sus alumnos. Por tanto, los limitados ingresos económicos que perciben, la necesidad de realizar dos o más actividades laborales, la condición socioeconómica en la que se encuentran -que en determinados casos llegan a la pobreza y pobreza extrema- y las respuestas insuficientes del Estado en la búsqueda de su bienestar, describen un entorno poco favorable para el logro de su realización personal y profesional.

Lo anteriormente mencionado conduce a buscar la relación entre el entorno del maestro (caracterizado por las presiones sociales y económicas antes descritas como condiciones estresantes) y las características individuales que poseen para afrontar dichas dificultades, ya que como se sabe, cuando el afrontamiento en las relaciones humanas es inadecuado se puede asociar a sentimientos de insatisfacción, ansiedad, desmotivación e incluso de hostilidad encubierta. De ello se desprende la necesidad de conocer aquellos factores que podrían inhibir su participación saludable, adecuada y a la vez creativa, en los ambientes educativos. El presente estudio pretende responder a las siguientes interrogantes:

1. ¿Qué características, en cuanto a los recursos de afrontamiento al estrés, presentan los docentes de colegios estatales de Lima Metropolitana?

2. ¿Qué características, en cuanto a las estrategias de afrontamiento, presentan los docentes de colegios estatales de Lima Metropolitana?

3. ¿Qué relación existe entre los recursos de afrontamiento y estrategias de afrontamiento al' estrés en docentes de colegios estatales de Lima Metropolitana?

\section{OBJETIVOS}

1. Analizar los recursos personales y las estrategias de afrontamiento al estrés en docentes de colegios estatales de' Lima Metropolitana.

2. Comparar ambas variables con relación a los niveles de enseñanza (Inicial, Primaria y Secundaria).

3. Analizar la correlación entre las variables recursos personales y estrategias de afrontamiento al estrés.

\section{HIPÓTESIS}

$\mathrm{H}_{1}$ : Existen diferencias estadísticamente significativas en los recursos de afrontamiento al estrés entre los docentes de centros escolares estatales de Lima Metropolitana de diferentes niveles de enseñanza (Inicial, Primaria y Secundaria).

$\mathrm{H}_{2}$ Existen diferencias significativas en las estrategias de afrontamiento entre los docentes de centros escolares estatales de Lima Metropolitana de diferentes de niveles de 
enseñanza (Inicial, Primaria, Secundaria).

$\mathrm{H}_{3}$ : Existe una correlación positiva entre las variables recursos personales y estrategias de afrontamiento al estrés en docentes de centros escolares estatales de Lima Metropolitana.

\section{METODO}

\section{Diseño de la Investigación}

Corresponde al descriptivo comparativo y correlacional, en el que se trata de describir y luego comparar por separado los recursos personales y las estrategias de afrontamiento según los niveles de enseñanza. De igual forma, se busca establecer la relación entre ambas variables de estudio.

\section{Muestra}

Está conformada por 546 docentes de centros educativos estatales de la zona de Lima Metropolitana (161 hombres y 385 mujeres), pertenecientes a los tres niveles de enseñanza: Inicial, Primaria y secundaria (ver Tab1a 1) en la que se considera también como variable el tiempo de servicio en la docencia (ver Tabla 2).

Los docentes fueron seleccionados a través de un muestreo no probabilístico, de tipo intencional, debido básicamente a las dificultades presentadas en la aplicación de las pruebas ya que al llegar a los centros educativos se evidenciaron actitudes de desconfianza, acompañadas de cierto tono de suspicacia de parte de los docentes, derivado posiblemente del momento político que acontecía en el país y de las acciones que se venían realizando desde el Ministerio de Educación, como por ejemplo la evaluación de los docentes a través de las USEs para observar la calidad de la enseñanza, así como la evaluación para los nombramientos.

Tabla 1: Descripción de la muestra por especialidad

\begin{tabular}{|c|c|c|}
\hline ESPECIALIDAD & $f$ & $\%$ \\
\hline INICIAL & 90 & 16.5 \\
PRIMARIA & 110 & 20.1 \\
SECUNDARIA & 346 & 63.4 \\
\hline TOTAL & 546 & 100 \\
\hline
\end{tabular}

Tabla 2: Descripción de la muestra por tiempo de servicio

\begin{tabular}{lll}
\hline TIEMPO DE SERVICIO (en años) & $f$ & $\%$ \\
\hline $0-5$ & 129 & 23,6 \\
$5-10$ & 146 & 26,7 \\
$10-15$ & 122 & 22,3 \\
$15-20$ & 98 & 17,9 \\
$20-25$ & 36 & 6,6 \\
$25-30$ & 11 & 2,0 \\
$35-40$ & 4 & 0,7 \\
\hline Total & 546 & 100.0
\end{tabular}




\section{Variables de estudio}

Variable Independiente: Centros escolares estatales, niveles de enseñanza y tiempo de servicio.

Variable Dependiente: Los resultados derivados de las pruebas sobre Recursos personales de afrontamiento y Estrategias de afrontamiento al estrés.

\section{Técnicas e instrumentos de recolección de datos}

Para la evaluación de las variables estudiadas se utilizaron los siguientes instrumentos psicológicos: Inventario de Recursos personales de afrontamiento de Hammer y Marting (1985), adaptado a nuestro medio por Valdez, N. (1999), como tema de tesis en la Pontificia Universidad Católica del Perú, el cual consta de 41 ítems tipo likert que evalúan cinco grupos de recursos personales:

1. Recursos Cognitivos

2. Recursos Sociales

3. Recursos Emocionales

4. Recursos Espirituales o Filosóficos

5. Recursos Físicos.

El autor considera los siguientes parámetros para la interpretación de los resultados:

\begin{tabular}{|l|c|c|c|c|c|c|}
\hline Parámetros & Recursos & Recursos & Recursos & Recursos & Recursos & Recursos \\
\hline $\begin{array}{l}\text { Bajos } \\
\text { recursos }\end{array}$ & $0-15$ & $0-20$ & $0-8$ & $0-13$ & $0-8$ & $0-66$ \\
\hline $\begin{array}{l}\text { Recursos } \\
\text { intermedios }\end{array}$ & $16-18$ & $21-25$ & $9-10$ & $14-17$ & $9-10$ & $67-77$ \\
\hline $\begin{array}{l}\text { Recursos } \\
\text { superiores }\end{array}$ & $19-$ más & $26-$ más & $11-$ más & $18-$ más & $11-$ más & $78-$ más \\
\hline
\end{tabular}

Análisis psicométrico de la escala: Se utilizó la correlación ítem-test corregida y la consistencia interna se estableció mediante el coeficiente de confiabilidad alfa de Cronbach (ver Tabla 3).

\section{Tabla 3: Recursos de afrontamiento. Coeficiente de Confiabilidad}

\begin{tabular}{|l|c|}
\hline \multicolumn{1}{|c|}{ ESCALAS } & Alfa de Cronbach \\
\hline 1. Recursos cognitivos & .6302 \\
2. Recursos emocionales & .5321 \\
3. Recursos sociales & .5120 \\
4. Recursos espirituales / filosóficos & .4736 \\
5. Recursos físicos & .5991 \\
\hline
\end{tabular}

Cuestionario de Modos o estrategias de afrontamiento al estrés (COPE) elaborado por Carver, S. (1989) y adaptado en nuestro medio por Casuso, L. (1996) con estudiantes universitarios de Lima (escala reorganizada). Está conformado por 52 ítems de formato tipo likert, distribuidos en tres áreas de acuerdo a las estrategias de afrontamiento: a) Enfocado al problema; b) Enfocado a la emoción; y c) Enfocado a otros estilos propuestos. Estas áreas derivan en trece escalas que corresponde a las trece estrategias de afrontamiento 
propuestas por el autor:

1. Estrategias de afrontamiento enfocado al problema

- Afrontamiento activo

- Planificación

- Supresión de actividades competentes

- Postergación del afrontamiento

- Búsqueda de apoyo social por razones instrumentales

2.Estrategias de afrontamiento enfocado a la emoción

- Búsqueda apoyo social por razones emocionales

- Reinterpretación positiva y crecimiento

- Aceptación

- Negación

- Acudir a la religión

3. Otras estrategias propuestas

- Enfocar y liberar emociones

- Desentendimiento conductual

- Desentendimiento mental

Carver (1989) toma como base en la estructuración de la prueba, los conceptos desarrollados por Lázarus y Folkman en 1986 y emplea el término estilo de afrontamiento de manera análoga a estrategias de afrontamiento, tanto en su dimensión disposicional como situacional, el cual es citado por Casuso (1996); sin embargo,. Fernández-Abascal (1997), citado por Guevara (2001), plantea una diferencia entre los estilos de afrontamiento y estrategias de afrontamiento para asignarle al primero un carácter más disposicional en las personas, mientr.as que al segundo le asigna un significado más concreto, y así referirse a la acción misma que utiliza la persona en cada contexto.

\section{Análisis psicométrico de la escala:}

Se utilizó la correlación item-test corregida y la consistencia interna se estableció mediante el coeficiente de confiabilidad alfa de Cronbach (Ver Tabla $\mathrm{N}^{\circ} 4$ ).

Tabla 4: Modos o estrategias de afrontamiento. Coeficiente de Confiabilidad

\begin{tabular}{|l|l|}
\hline ESCALAS & Alfa de Cronbach \\
\hline 1. Afrontamiento activo & \\
2. Planificación & .4745 \\
3. Supresión de actividades competentes & .6905 \\
4. Postergación del afrontamiento & .6737 \\
5. Búsqueda de apoyo social por razones & .3821 \\
instrumentales & .7398 \\
6. Búsqueda apoyo social por razones & .7963 \\
emocionales & .6523 \\
7. Reinterpretación positiva y crecimiento & .5535 \\
8. Aceptación & .5136 \\
9. Negación & .7811 \\
10.Acudir a la religión & .5937 \\
11.Enfocar y liberar emociones & .6600 \\
12.Desentendimiento conductual & .4428 \\
13.Desentendimiento mental & \\
\hline
\end{tabular}




\section{RESULTADOS}

Al realizar las comparaciones de los resultados de acuerdo a los niveles de enseñanza, se obtienen los siguientes hallazgos:

Tabla 5: Comparación de variables recursos y estrategias de afrontamiento al estrés en docentes de Inicial y Primaria

\begin{tabular}{|c|c|c|c|c|c|}
\hline \multirow[t]{2}{*}{ Áreas medias } & \multicolumn{2}{|c|}{ Inicial } & \multicolumn{2}{|c|}{ Primaria } & \multirow[b]{2}{*}{ " $t "$} \\
\hline & $\mathrm{X}$ & D.S & $\mathrm{X}$ & D.S & \\
\hline I. Recursos de afrontamiento & & & & & \\
\hline 1. Recursos cognitivos & 19.73 & 3.15 & 19.75 & 4.19 & 0.34 \\
\hline 2. Recursos emocionales & 18.26 & 3.73 & 18.09 & 3.61 & 0.33 \\
\hline 3.Recursos sociales & 8.19 & 1.78 & 8.50 & 1.96 & 1.16 \\
\hline 4.Recursos físicos & 8.27 & 2.80 & 8.60 & 3.37 & 0.74 \\
\hline 5.Recursos totales & 10.17 & 1.52 & 9.78 & 1.82 & 1.62 \\
\hline 6.Recursos totales & 64.61 & 5.94 & 64.53 & 6.61 & 0.09 \\
\hline II. Estrategias de afrontamiento & & & & & \\
\hline A. estrategias de afrontamiento que enfoca el problema & & & & & \\
\hline 1.Afrontamiento activo & 10.39 & 2.06 & 10.51 & 2.28 & 0.39 \\
\hline 2.Planificación & 11.60 & 2.58 & 11.55 & 2.48 & 0.14 \\
\hline 3.Supresión de actividades competentes & 9.28 & 2.49 & 9.20 & 2.54 & 0.22 \\
\hline 4.Poatergación del afrontamiento & 9.84 & 1.87 & 10.40 & 1.88 & $2.10^{*}$ \\
\hline 5.Busqueda de apoyo social por razones instrumentales & 10.27 & 2.56 & 9.98 & 2.54 & 0.80 \\
\hline B. Estrategias de afrontamiento que enfocan la emoción & & & & & \\
\hline 6.Busqueda de apoyo social por razones emocionales & 10.27 & .3 .02 & 9.85 & 3.18 & 0.95 \\
\hline 7.Reinterpretación positiva y crecimiento & 12.99 & 2.20 & 12.46 & 2.41 & 1.61 \\
\hline 8.Aceptación & 10.86 & 2.33 & 10.43 & 2.58 & 1.22 \\
\hline 9.Negación & 7.48 & 2.05 & 7.89 & 2.29 & 1.32 \\
\hline 10. Acudir a la religión & 12.01 & 2.55 & 11.47 & 3.01 & 1.35 \\
\hline C. Otras estrategias propuestas & & & & & \\
\hline 11. Enfocar y liberar emoción & 8.92 & 2.31 & 8.97 & 2.26 & 0.15 \\
\hline 12. Desentendimiento conductual & 6.23 & 1.97 & 6.78 & 2.25 & 1.82 \\
\hline 13. Desentendimiento mental & 7.87 & 2.21 & 8.18 & 2.31 & 0.96 \\
\hline
\end{tabular}

En cuanto a los Recursos de afrontamiento, si bien no se observan diferencias estadísticamente significativas entre los docentes de Inicial y Primaria (ver Tabla 5), sin embargo, de acuerdo a los parámetros descritos anteriormente por el autor, se puede afirmar que los puntajes de ambos grupos revelan la presencia de bajos recursos de afrontamiento, especialmente en las escalas recursos emocionales, sociales, espirituales / filosóficos y totales; un nivel intermedio en la escala de recursos físicos, y un nivel alto en la escala de recursos cognitivos. Esto evidencia un mayor uso y manejo del aspecto racional del docente, es decir, tienden a ser analíticos y reflexivos ante las situaciones estresantes.

En cuanto a las Estrategias de afrontamiento, se pueden observar diferencias estadísticamente significativas sólo en la escala Postergación del Afrontamiento $(t=2.10$, $P<.05)$, en la cual la media mayor la obtienen los docentes de Primaria. Esto estaría revelando que dichos docentes evidencian una mayor tolerancia que los docentes de Inicial para actuar sobre los problemas que se le presenten, ellos prefieren esperar el momento oportuno para actuar sin precipitarse.

Si bien es cierto que en las otras escalas no se observan diferencias significativas entre ambos grupos de comparación, sin embargo, habría que destacar aquellos resultados que 
sobresalen de la media alcanzada por la muestra evaluada, así vemos que la escala Reinterpretación positiva y crecimiento se encuentra en un nivel alto en ambos grupos, lo que estaría evidenciando la capacidad que tienen los docentes de Inicial y Primaria para evaluar en forma positiva los problemas que les suscitan, brindándole connotaciones favorables a la experiencia misma.

En cuanto a la escala Desentendimiento conductual, ambos grupos alcanzan el nivel más bajo en este rubro, lo que significa que dichos docentes se caracterizan por brindar sus mayores esfuerzos para afrontar al estresor y así alcanzar sus metas, es decir, son conscientes de los problemas u obstáculos que los limitan y lejos de «desentenderse» del problema, lo enfrentan a través de sus acciones o «conductas».

En términos generales, no se encuentran mayores diferencias entre las respuestas obtenidas por los docentes de Inicial y Primaria.

Tabla 6: Comparación de variables recursos y estrategias de afrontamiento al estrés en docentes de Inicial y Secundaria

\begin{tabular}{|l|l|l|l|l|l|}
\hline \multirow{2}{*}{ Áreas medias } & \multicolumn{2}{|c|}{ Inicial } & \multicolumn{2}{|c|}{ Secundaria } & \\
\cline { 2 - 6 } & $\mathrm{X}$ & $\mathrm{D} . \mathrm{S}$ & $\mathrm{X}$ & $\mathrm{D} . \mathrm{S}$ & "t" \\
\hline I. Recursos de afrontamiento & & & & & \\
1. Recursos cognitivos & 19.73 & 3.15 & 20.80 & 3.39 & $2.71^{*}$ \\
2. Recursos emocionales & 18.26 & 3.73 & 18.03 & 3.21 & $0.58^{*}$ \\
7.Recursos sociales & 8.19 & 1.78 & 7.95 & 1.85 & 1.10 \\
8.Recursos físicos & 8.27 & 2.80 & 8.32 & 3.47 & 0.13 \\
9.Recursos totales & 10.17 & 1.52 & 10.30 & 1.73 & 0.65 \\
10.Recursos totales & 64.61 & 5.94 & 65.40 & 6.52 & 1.04 \\
\hline II. Estrategias de afrontamiento & & & & & \\
A. Estrategias de afrontamiento que enfoca el problema & & & & & \\
1.Afrontamiento activo & 10.39 & 2.06 & 10.51 & 2.09 & 2.60 \\
2.PlanificaciónZ & 11.60 & 2.58 & 11.55 & 2.36 & $2.14 *$ \\
3.Supresión de actividades competentes & 9.28 & 2.49 & 9.20 & 2.63 & 1.04 \\
4.Poatergación del afrontamiento & 9.84 & 1.87 & 10.40 & 2.15 & 2.26 \\
5.Busqueda de apoyo social por razones instrumentales & 10.27 & 2.56 & 10.40 & 2.79 & 0.40 \\
B. Estrategias de afrontamiento que enfocan la emoción & & & & & \\
6.Busqueda de apoyo social por razones emocionales & 10.27 & 3.02 & 10.01 & 2.97 & 0.74 \\
7.Reinterpretación positiva y crecimiento & 12.99 & 2.20 & 13.15 & 2.13 & 0.63 \\
8.Aceptación & 10.86 & 2.33 & 10.53 & 2.41 & 1.17 \\
9.Negación & 7.48 & 2.05 & 7.34 & 2.12 & 0.56 \\
10. Acudir a la religión & 12.01 & 2.55 & 10.89 & 3.35 & $2.96^{*}$ \\
C. Otras estrategias propuestas & & & & & \\
11. Enfocar y liberar emoción & 8.92 & 2.31 & 8.48 & 2.31 & 1.61 \\
12. Desentendimiento conductual & 6.23 & 1.97 & 6.00 & 2.02 & 0.97 \\
13. Desentendimiento mental & 7.87 & 2.21 & 7.95 & 2.15 & 0.31 \\
\hline
\end{tabular}

En la Tabla 6 se observan los resultados alcanzados por los docentes de Inicial y Secundaria, entre quienes se establecen los niveles de comparación, en primer lugar, sobre los Recursos personales de afrontamiento, donde se presentan diferencias significativas en el factor Recursos cognitivos a favor de los docentes de Secundaria, lo que significaría que este grupo maneja mejor dichos recursos, sin embargo, también se debe destacar que ambos grupos alcanzan niveles altos en esta escala en función a las otras, lo que representa un mejor manejo del aspecto racional durante la toma de decisiones al enfrentar situaciones difíciles. A esto se debe agregar que tanto los docentes de Inicial como los de Secundaria, alcanzan un nivel intermedio en cuanto a los Recursos físicos que utilizan al afrontar situaciones estresantes, lo que significaría que dichos grupos reconocen la importancia de 
fomentar en ellos ciertas conductas orientadas al bienestar y salud físico. En cuanto a los resultados obtenidos en las otras escalas, se traslucen la presencia de bajos recursos emocionales, sociales, espirituales o filosóficos y totales.

Si bien existe una preferencia en el uso de la racionalidad y la reflexión para afrontar las situaciones difíciles que se les presente, esta tiene un carácter más individual que colectivo, ya que se observan dificultades para participar en redes sociales como complemento del afrontamiento. En este sentido, se tendría que ver cuáles son las características que tiene dicha reflexión, ya que una forma de manifestarse sería a través de acciones constructivas, canalizadas en el campo de la creatividad y la promoción del cambio; otra forma de manifestación se daría de manera negativa en el campo de las relaciones interpersonales, en la que el docente podría asumir una actitud cuestionadora, y como es obvio, esto afectaría a la convivencia, caracterizado por un tono de desconfianza.

En segundo lugar, en cuanto a las Estrategias o estilos de afrontamiento, se observan diferencias significativas sobre todo en las escalas que pertenecen al grupo de estrategias que enfocan el problema:

Afrontamiento activo, planificación y postergación del afrontamiento, con una media mayor a favor de los docentes de Secundaria, lo que estaría evidenciando que dichos docentes prefieren buscar la solución a sus problemas de forma directa, centrando su atención en la dificultad o en el elemento estresor, el cual se encuentra fuera de ellos mismos. Esto no quiere decir que los docentes de Inicial no dirijan la atención y los esfuerzos a encontrar la solución a sus problemas, sólo que ellos muestran una mayor preferencia por las estrategias que enfocan la emoción, que les permitan acudir a la religión, por lo que la media alcanzada por este grupo en esta escala es significativamente mayor en comparación a los docentes de Secundaria.

Esto puede entenderse en la medida que ambos grupos de docentes (Inicial y Secundaria) trabajan con alumnos cuyas características y necesidades educativas son totalmente diferentes: mientras que los docentes de Inicial buscan generar espacios afectivos y poco traumáticos para los niños que por primera vez se separan por tiempos prolongados de sus familias, centrando su labor en el aspecto socioafectivo del menor, los docentes del nivel Secundario, por el contrario, buscan generar autonomía en los adolescentes, responsabilidad en las acciones que realizan y en la toma de decisiones sobre su propia vida. Esto hace que las conductas que desarrollen los docentes de ambos grupos sean también diferentes, y por lo tanto, las estrategias que utilicen para abordar los problemas escolares tengan otro matiz: mientras que los docentes de Inicial denotan una conducta cálida, empática, de acercamiento y preocupación por el bienestar del menor, desarrollando estrategias centradas en la emoción, sobretodo en fa espiritualidad, tratando de transmitirlas a sus alumnos, los docentes de Secundaria prefieren establecer una relación basada en la autoridad, en la que cumplen la función de enseñar y dejan a los alumnos la responsabilidad de aprender. Pese a que las nuevas orientaciones pedagógicas sugieren el establecimiento de relaciones afectivas y empáticas que favorezcan el logro de capacidades en los alumnos, los docentes de Secundaria aún tienen dificultad para plasmar dichos principios en las aulas de clase.

Se puede mencionar como ejemplo, el manejo de la disciplina en el aula, en la que si el alumno <<no se porta bien», es decir, conversa en clase, hace «bulla» o realiza otras acciones no vinculadas a la sesión de aprendizaje, es sancionado con la expulsión del aula, desaprobado o simplemente se le coloca una nota de mala conducta. De esta forma, los 
docentes prefieren utilizar estrategias de afrontamiento centrados en el problema, ejecutando acciones directas a fin de acabar con el elemento estresor.

Tabla 7: Comparación de variables recursos y estrategias de afrontamiento al estrés en docentes de Primaria y Secundaria

\begin{tabular}{|l|l|l|l|l|l|}
\hline \multicolumn{1}{|c|}{ Áreas medias } & \multicolumn{2}{c|}{ Primaria } & \multicolumn{2}{c|}{ Secundaria } & \\
\cline { 2 - 6 } & $\mathrm{X}$ & $\mathrm{D} . \mathrm{S}$ & $\mathrm{X}$ & $\mathrm{D} . \mathrm{S}$ & "t" \\
\hline 1. Recursos de afrontamiento & & & & & \\
2. Recursos cognitivos & 19.75 & 4.19 & 20.80 & 3.39 & $3.17^{*}$ \\
3. Recursos emocionales & 18.09 & 3.61 & 18.03 & 3.21 & 0.1655 \\
4. Recursos sociales & 8.50 & 1.96 & 7.95 & 1.85 & $2.68^{*}$ \\
5. Recursos físicos & 8.60 & 3.37 & 8.32 & 3.47 & 0.7422 \\
6. Recursos totales & 9.78 & 1.82 & 10.30 & 1.73 & $2.71^{*}$ \\
7. Recursos totales & 64.53 & 6.61 & 65.40 & 6.52 & 1.22 \\
\hline II. Estrategias de afrontamiento & & & & & \\
A. Estrategias de afrontamiento que enfoca el problema & & & & & \\
1.Afrontamiento activo & 10.51 & 2.28 & 11.03 & 2.09 & $2.23^{*}$ \\
2.Planificación & 11.55 & 2.48 & 12.21 & 2.36 & $2.52^{*}$ \\
3.Supresión de actividades competentes & 9.20 & 2.54 & 9.60 & 2.63 & 1.40 \\
4.Poatergación del afrontamiento & 10.40 & 1.88 & 10.40 & 2.15 & 0.00 \\
5.Busqueda de apoyo social por razones instrumentales & 9.98 & 2.54 & 10.40 & 2.79 & 1.40 \\
B. Estrategias de afrontamiento que enfocan la emoción & & & & & \\
6.Busqueda de apoyo social por razones emocionales & 9.85 & 3.18 & 10.01 & 2.97 & 0.48 \\
7.Reinterpretación positiva y crecimiento & 12.46 & 2.41 & 13.15 & 2.13 & $2.87^{*}$ \\
8.Aceptación & 10.43 & 2.58 & 10.53 & 2.41 & 0.37 \\
9.Negación & 7.89 & 2.29 & 7.34 & 2.12 & $2.32^{*}$ \\
10. Acudir a la religión & 11.47 & 3.01 & 10.89 & 3.35 & 1.62 \\
C. Otras estrategias propuestas & & & & & \\
11. Enfocar y liberar emoción & 8.97 & 2.26 & 8.48 & 2.31 & 1.95 \\
12. Desentendimiento conductual & 6.78 & 2.25 & 6.00 & 2.02 & $3.43^{*}$ \\
13. Desentendimiento mental & 8.18 & 2.31 & 7.95 & 2.15 & 0.96 \\
\hline P P .05
\end{tabular}

$* \mathrm{P}<.05$

Al analizar y comparar los resultados obtenidos por los docentes de primaria y secundaria (ver Tabla 07) se puede observar que presentan diferencias significativas en las escalas de Recursos de afrontamiento cognitivos y físicos, a favor de los docentes de Secundaria, lo que evidencia una mayor disposición reflexiva de parte de estos docentes ante situaciones estresoras; en cambio, los docentes de Primaria demuestran poseer mayores Recursos sociales que los docentes de Secundaria, lo que revela una mayor disposición a actuar en redes sociales cuando se presentan dificultades.

En cuanto a las Estrategias de afrontamiento, se pueden observar diferencias significativas en las escalas afrontamiento activo, planificación y reinterpretación positiva y crecimiento, en las que la media es mayor en el grupo de docentes de Secundaria. Esto se puede interpretar como la preferencia -del grupo mencionado- en el uso de estrategias centradas en el problema, las que se relacionan con una planificación y ejecución de acciones directas dirigidas a evitar al estresor o aminorar sus efectos; además, es necesario agregar que han desarrollado una mejor capacidad para evaluar los problemas de forma positiva, otorgándole connotaciones favorables, en comparación con los docentes de Primaria.

Por otro lado, en cuanto a los resultados obtenidos en las escalas negación y 
desentendimiento conductual, también se observan diferencias significativas, pero en este caso, a favor de los docentes de Primaria. Sin embargo, es bueno reflexionar sobre los resultados globales ya que en ambas escalas los dos grupos han obtenido puntajes bajos, por lo tanto, aquellos indicadores que podrían interpretarse como una mayor disposición de los docentes de Primaria a negar las situaciones estresoras y disminuir el esfuerzo para afrontarlo en comparación con los docentes de Secundaria, es relativo en la medida que los resultados estadísticos marcan una diferencia significativa, pero los resultados globales refieren que son las estrategias menos utilizadas por ambos grupos de docentes.

Tabla 8: Comparación de variables recursos y estrategias de afrontamiento al estrés en docentes de acuerdo al tiempo de servicio

\begin{tabular}{|c|c|c|c|c|c|}
\hline \multirow[t]{2}{*}{ FACTORES MEDIDOS } & \multicolumn{2}{|c|}{$\begin{array}{l}11 \text { años } \\
n=271\end{array}$} & \multicolumn{3}{|c|}{$\begin{array}{l}10 \text { años } \\
\mathrm{n}=275\end{array}$} \\
\hline & $\mathrm{X}$ & D.S. & $X$ & D.S. & "t $\mathrm{t} "$ \\
\hline \multicolumn{6}{|l|}{ I. RECURSOS DE AFRONTAMIENTO } \\
\hline 1. Recursos Cognitivos & 20.28 & 3.65 & 20.47 & 3.49 & -0.63 \\
\hline 2. Recursos Emocionales & 18.08 & 3.53 & 18.08 & 3.23 & 0.01 \\
\hline 3. Recursos Sociales & 8.08 & 1.86 & 8.12 & 1.89 & -0.22 \\
\hline 4. Recurso Espiritual Filosófico & 7.83 & 336 & 8.90 & 3.24 & $-3.76 *$ \\
\hline 5. Recursos Físicos & 10.13 & 1.72 & 10.21 & 1.73 & -0.53 \\
\hline 6. Recursos Totales & 64.40 & 6.76 & 65.77 & 6.06 & $-2.49 *$ \\
\hline \multicolumn{6}{|l|}{ Il. ESTILOS DE AFRONTAMIENTO } \\
\hline \multicolumn{6}{|l|}{ A. Estrategias de afrontamiento que enfoca el problema } \\
\hline 1. Afrontamiento Activo & 11.01 & 2.20 & 10.64 & 2.06 & $2.05 *$ \\
\hline 2. Planificación & 12.18 & 2.38 & 11.78 & 2.48 & 1.90 \\
\hline 3. Supresión de actividades competentes & 9.34 & 2.63 & 9.59 & 2.55 & -1.14 \\
\hline 4. Postergación del afrontamiento & 10.28 & 2.17 & 1033 & 1.96 & -033 \\
\hline 5. Búsqueda de apoyo social por razón. instrumentales & 10.15 & 2.75 & 10.43 & 2.66 & -1.18 \\
\hline \multicolumn{6}{|l|}{ B. Estrategias de afrontamiento que enfoca la emoción } \\
\hline 6. Búsqueda de apoyo social por razones emocionales & 9.94 & 3.04 & 10.09 & 3.00 & -0.58 \\
\hline 7. Reinterpretación positiva y crecimiento & 12.96 & 2.23 & 13.01 & 2.20 & -0.23 \\
\hline 8. Aceptación & 10.63 & 2.63 & 10.49 & 2.22 & 0.67 \\
\hline 9. Negación & 7.55 & 2.25 & 7.41 & 2.05 & 0.75 \\
\hline 10. Acudir a la religión & 11.47 & 3.18 & 10.92 & 3.18 & $2.03 *$ \\
\hline \multicolumn{6}{|l|}{ C. Otras estrategias propuestas } \\
\hline 11. Enfocar y liberar emoción & 8.82 & 2.47 & 8.49 & 2.13 & 1.63 \\
\hline 12. Desentendimiento conductual & 6.36 & 2.17 & 6.03 & 1.96 & 1.88 \\
\hline 13. Desentendimiento mental & 7.87 & 2.32 & 8.09 & 2.05 & -1.19 \\
\hline
\end{tabular}

Para realizar un análisis comparativo de los resultados obtenidos de acuerdo al tiempo de servicio (ver Tabla 8) se agrupó a la muestra evaluada en: Grupo A (docentes con once años de servicio o más) y Grupo B (docentes con diez años de servicio o menos). De esta forma, tenemos los siguientes alcances:

En cuanto a los Recursos de afrontamiento, se observan diferencias significativas en las escalas Recurso espiritual/filosófico y recursos totales, cuyas medias son mayores en el grupo B, es decir, en los docentes que tienen diez años de servicio o menos. Esto significa que los docentes con menos años de servicio prefieren realizar acciones guiadas por valores estables y consistentes; además, cuentan con mayores recursos totales de afrontamiento que los docentes con mayor antigüedad en la profesión. Sin embargo, al realizar un análisis de acuerdo a los parámetros presentados por el autor, se puede apreciar que los resultados en ambas escalas son bajos, lo que significa que, independientemente de 
los años de servicio, los docentes no presentan adecuados recursos personales que les permita enfrentar adecuadamente las situaciones estresantes.

En cuanto a las estrategias de afrontamiento, se observan diferencias significativas en las escalas Afrontamiento activo y acudir a la religión, cuyas medias son mayores en los docentes del grupo A, es decir, en los docentes con once años de servicio o más. Esto podría significar que dichos docentes gozan de una mayor seguridad para actuar en forma más activa ante situaciones estresantes, probablemente el hecho de estar nombrados en el estamento docente, les dé la confianza suficiente como para decir y actuar de acuerdo a sus ideas y principios; de igual forma evidencian una mayor tendencia a acudir a la religión ante situaciones estresantes, como una forma de aliviar la tensión.

Tabla 9: Correlación entre los Recursos de Afrontamiento y las Estrategias de Afrontamiento

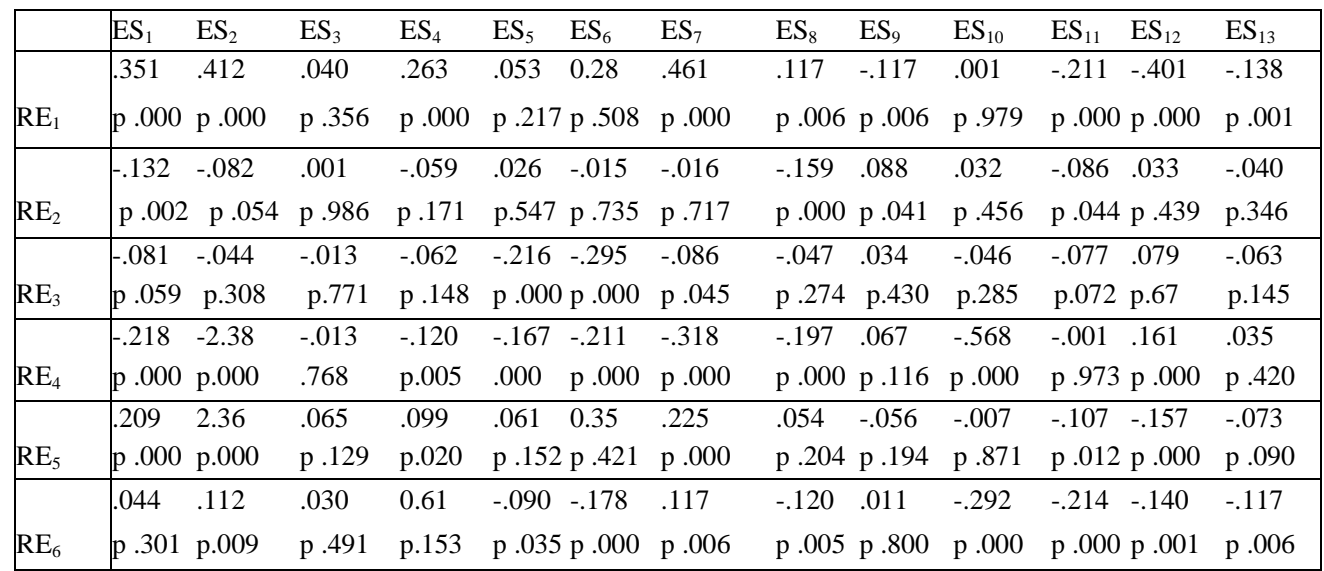

Tabla 10: Correlación entre los Recursos de Afrontamiento y las Estrategias de Afrontamiento

\begin{tabular}{|c|c|c|c|c|c|c|c|c|c|c|c|c|c|}
\hline & $\mathrm{ES}_{1}$ & $\mathrm{ES}_{2}$ & $\mathrm{ES}_{3}$ & $\mathrm{ES}_{4}$ & $\mathrm{ES}_{5}$ & $\mathrm{ES}_{6}$ & $\mathrm{ES}_{7}$ & $\mathrm{ES}_{8}$ & $\mathrm{ES}_{9}$ & $\mathrm{ES}_{10}$ & $\mathrm{ES}_{11}$ & $\mathrm{ES}_{12}$ & $\mathrm{ES}_{13}$ \\
\hline & .351 & .412 & & .263 & & & .461 & .117 & -.117 & & -.211 & -.401 & -.138 \\
\hline $\mathrm{RE}_{1}$ & P.000 & p .000 & & p .000 & & & p .000 & p.006 & p.006 & & p. .000 & p. .000 & p.001 \\
\hline & -.132 & & & & & & & -.159 & .088 & & -.086 & & \\
\hline $\mathrm{RE}_{2}$ & p .00 & & & & & & & p. .000 & p.041 & & p.044 & & \\
\hline $\mathrm{RE}_{3}$ & & & & & $\begin{array}{l}-.216 \\
\text { p } .000\end{array}$ & $\begin{array}{l}-.295 \\
\text { p .000 }\end{array}$ & $\begin{array}{l}-.086 \\
\text { p } .045\end{array}$ & & & & & & \\
\hline $\mathrm{RE}_{4}$ & $\begin{array}{l}-.218 \\
\mathrm{p} .000\end{array}$ & $\begin{array}{l}-2.38 \\
\text { p.000 }\end{array}$ & & $\begin{array}{l}-.120 \\
\text { p.005 }\end{array}$ & $\begin{array}{l}-.167 \\
.000\end{array}$ & $\begin{array}{l}-.211 \\
\text { p .000 }\end{array}$ & $\begin{array}{l}-.318 \\
\text { p } .000\end{array}$ & $\begin{array}{l}-.197 \\
\text { p } .000\end{array}$ & & $\begin{array}{l}-.568 \\
\text { p .000 }\end{array}$ & & $\begin{array}{l}.161 \\
\text { p } .000\end{array}$ & \\
\hline $\mathrm{RE}_{5}$ & $\begin{array}{l}.209 \\
\mathrm{p} .000\end{array}$ & $\begin{array}{l}2.36 \\
\text { p.000 }\end{array}$ & & $\begin{array}{l}.099 \\
\text { p.020 }\end{array}$ & & & $\begin{array}{l}.225 \\
\text { p } .000\end{array}$ & & & & $\begin{array}{l}-.107 \\
\text { p.012 }\end{array}$ & $\begin{array}{l}-.157 \\
\text { p } .000\end{array}$ & \\
\hline $\mathrm{RE}_{6}$ & & $\begin{array}{l}.112 \\
\text { p .009 }\end{array}$ & & & $\begin{array}{l}-.090 \\
\text { p .035 }\end{array}$ & $\begin{array}{l}-.178 \\
\text { p .000 }\end{array}$ & $\begin{array}{l}.117 \\
\text { p .006 }\end{array}$ & $\begin{array}{l}-.120 \\
\text { p.005 }\end{array}$ & & $\begin{array}{l}-.292 \\
\text { p .000 }\end{array}$ & $\begin{array}{l}-.214 \\
\text { p. } .000\end{array}$ & $\begin{array}{l}-.140 \\
\text { p .001 }\end{array}$ & $\begin{array}{l}-.117 \\
\text { p .006 }\end{array}$ \\
\hline
\end{tabular}

Pearson N=546

$\mathbf{P}<.05$ 
En las tablas 9 y 10, se aprecian los siguientes resultados:

RECURSO COGNITIVO: Se observa una correlación positiva con las escalas afrontamiento activo, planificación, postergación del afrontamiento, reinterpretación positiva y crecimiento y aceptación; además de una correlación negativa con las escalas negación, enfocar y liberar emociones, desentendimiento conductual y desentendimiento mental.

RECURSOS EMOCIONALES: Se observa una correlación positiva con la escala negación y una correlación negativa con las escalas afrontamiento activo, aceptación y enfocar y liberar emociones,

RECURSOS SOCIALES: Presenta una correlación negativa con las escalas búsqueda de apoyo social por razones instrumentales, búsqueda de apoyo social por razones emocionales y reinterpretación positiva y crecimiento,

RECURSOS ESPIRITUALES FILOSÓFICOS: Presenta una correlación positiva con la escala desentendimiento conductual y una correlación negativa con las escalas afrontamiento activo, planificación, postergación del afrontamiento, búsqueda de apoyo social por razones instrumentales, búsqueda de apoyo social por razones emocionales, reinterpretación positiva y crecimiento, aceptación y acudir a la religión,

RECURSOS FÍSICOS: Presenta una correlación positiva con las escalas afrontamiento activo, planificación, postergación del afrontamiento, reinterpretación positiva y crecimiento, y una correlación negativa con las escalas enfocar y liberar emociones y desentendimiento conductual.

RECURSOS TOTALES: Presenta una correlación positiva con las escalas planificación y reinterpretación positiva y crecimiento y una correlación negativa con las escalas búsqueda de apoyo social por razones instrumentales, búsqueda de apoyo social por razones emocionales, aceptación, acudir a la religión, enfocar y liberar emociones, desentendimiento conductual y desentendimiento mental. 


\section{DISCUSIÓN}

En cuanto al factor Recursos de Afrontamiento, el cual hace referencia a la capacidad o potencialidad que posee la persona para manejar los elementos estresores (Lazarus y Folkman, 1986), se observan indicadores de la presencia de recursos cognitivos superiores en los docentes de los tres niveles de enseñanza, lo cual evidencia un mayor uso del aspecto racional y lógico al elegir las estrategias más adecuadas para enfrentar los estresores. En segundo lugar, se encuentran los recursos físicos, como recursos intermedios también en los docentes de los tres niveles de enseñanza.

$\mathrm{Al}$ respecto, se puede señalar que una de las características que sobresale en los docentes es la capacidad para racionalizar al momento de evaluar sus experiencias estresantes y al momento de elegir determinada estrategia de afrontamiento. Esto puede ser positivo -ya que la evaluación de por sí cuenta con un componente cognitivo-, sin embargo, al no encontrarse otros recursos que pudiesen influir en dicha evaluación, por ejemplo, recursos sociales o espirituales, se puede inferir que dichas estrategias o alternativas de solución que encuentran a sus problemas no estén acompañadas de habilidades sociales y de recursos emocionales, que les permita alcanzar una correcta canalización.

Además, se tiene que, en términos generales, los docentes de los tres niveles de enseñanza no gozan de suficientes recursos de afrontamiento que les permitan responder de forma adecuada a las situaciones de estrés.

Al respecto hay que considerar lo manifestado por Zeidner y Hammer (1992) quienes consideran que las personas con bajos recursos de afrontamiento al estrés son sujetos vulnerables para afrontar una crisis mayor y constitucionalmente frágiles para manejar eventos estresores, no optan por un afrontamiento aproximativo, sino más bien por un afrontamiento evitativo y por lo tanto experimentan muchas dificultades emocionales.

En cuanto a las diferencias por niveles de enseñanza, no se encuentran diferencias significativas entre los docentes de Inicial y Primaria, pero sí entre los de Primaria y Secundaria. Esto se puede entender en la medida que los docentes de Inicial y Primaria son profesionales preparados para la atención a «niños», quienes dependen de los adultos; mientras que los de Secundaria se preparan para la atención de «adolescentes», a quienes deben enseñar a asumir con responsabilidad sus acciones (en algunos casos, laborales) y no depender de otros.

En cuanto a los Modos o estrategias de afrontamiento que utilizan los docentes para solucionar sus problemas o situaciones de estrés, se evidencian diferencias significativas sobre todo entre los docentes de Inicial y Primaria con los de Secundaria.

En este sentido, los docentes de Secundaria denotan un perfil que apunta al uso de estrategias de afrontamiento centradas en el problema, en las que utilizan preferentemente el afrontamiento activo al estresor y la planificación de sus estrategias. Esto se relaciona con los recursos cognitivos de afrontamiento que también sobresalen en ellos, ya que al evaluar en forma racional una situación estresante, prefieren utilizar estrategias también racionales que se dirijan a apartar, aminorar o evitar el estresor, por lo general, exterior a ellos. Pero, además, se debe agregar la capacidad que tienen para reinterpretar en forma positiva el problema, dándole connotaciones favorables, pese a que no necesariamente las soluciones racionales que encuentren sean las más adecuadas. 
En este sentido, encontramos ligeras diferencias con respecto a los docentes de Inicial y Primaria. Ellos -a diferencia de los docentes de Secundaria- prefieren utilizar estrategias centradas en la emoción, como son la reinterpretación positiva de los problemas y el acudir a la religión, con la intención de reducir la tensión. Además, también hacen uso de la planificación a través de la organización de sus acciones. Como ya se señaló anteriormente, el tener a su cargo a niños que aún dependen de los adultos, hace que asuman una mayor responsabilidad sobre sus acciones, y por lo tanto, planifiquen mejor aquellas estrategias que utilizarán para enfrentar las situaciones estresantes. Esta podría ser la razón por la que al asumir posturas firmes ante los problemas generales de los docentes, son los de Secundaria los que asumen acciones más directas y frontales que los de Inicial y Primaria.

\section{CONCLUSIONES}

De acuerdo al análisis realizado sobre la base de los resultados obtenidos en las pruebas, se puede concluir lo siguiente:

1. Se presentan diferencias estadísticamente significativas entre los docentes de Inicial y Secundaria en la escala Recursos Cognitivos, a favor de los docentes de Secundaria; y entre los docentes de Primaria y Secundaria en las escalas Recursos Cognitivos, Físicos (a favor de los docentes de Secundaria) y Sociales (a favor de los docentes de Primaria).

2. No se presentan diferencias estadísticamente significativas entre los docentes de Inicial y Primaria en cuanto a los recursos de afrontamiento al estrés.

3. Los recursos de afrontamiento más utilizados por los docentes de centros escolares estatales son los Recursos Cognitivos.

4.Se presenta diferencias estadísticamente significativas entre los docentes de Inicial y Primaria en la escala Postergación del afrontamiento, a favor de los docentes de Primaria; y entre los docentes de Inicial y Secundaria, en las escalas Afrontamiento activo, planificación, postergación del afrontamiento (a favor de los de Secundaria) y acudir a la religión (a favor de los de Inicial).

5. Se presentan diferencias estadísticamente significativas entre los docentes de Primaria y Secundaria en las escalas Afrontamiento activo, planificación, re interpretación positiva y crecimiento (a favor de los docentes de Secundaria), negación y desentendimiento conductual (a favor de los docentes de Primaria).

6. Los docentes de Inicial y Primaria prefieren utilizar como estrategias de afrontamiento, la planificación, reinterpretación positiva y crecimiento y acudir a la religión; mientras que los docentes de Secundaria prefieren utilizar las estrategias de afrontamiento activo, planificación y reinterpretación positiva y crecimiento.

7. Se observa correlación entre la variable recursos personales y la variable estrategias de afrontamiento al estrés, en algunos casos la correlación es positiva y en otras la correlación es negativa. 


\section{REFERENCIAS BIBLIOG RÁFICAS}

Bjorck, P. y Cohen, L. (1993). Afrontamiento a situaciones estresantes de pérdida, amenaza y reto. Journal of Social and Clinical Psychology

Buendía, J. (1993). Estrés y Psicopatología. Madrid: Editorial Pirámide.

Burns y Nole (1991). Estilos de afrontamiento en pacientes depresivos. Journal of Consulting and Clinical Psychology.

Casuso, L. (1996). Adaptación de la prueba de COPE sobre estilos de afrontamiento en grupo de estudiantes universitarios de Lima. Tesis. Lima: PUCP.

Guevara, G. (2001). Estilo de afrontamiento al estrés en pacientes drogadependientes. Revista de Investigación en Psicología. UNMSM. 4(1): 53-65.

Salazar, V. (1993). Relaciones entre estilos de afrontamiento al estrés y las dimensiones de Personalidad: Neuroticismo y Extraversión. Tesis. Lima: UPCH.

Lazarus, R. y Folkman, S. (1986). Estrés y procesos cognitivos. Barcelona: Ediciones Martinez Roca.

Moscoso, M. (1998). Psicología de la Salud: Hacia una nueva ciencia de la conducta. Revista de Psicología. UNMSM. II(I).

Moscoso, M. (1998). Estrés, salud y emociones: Estudio de la ansiedad, cólera y hostilidad. Revista de Psicología. UNMSM. III(3).

Moscoso, M. (1998). Medición de la expresión del cólera y hostilidad. Revista de Psicología. UNMSM. III(4).

Rodríguez, J. (1995). Psicología Social de la Salud. Madrid: Síntesis. 1ra. Edición. Spielberger, Ch. y Reheiser, E. (1998).

Encuesta de estrés laboral: Diferencias de género en la medición del estrés ocupacional. Revista de Psicología. UNMSM. III(3).

Spielberger, Ch. y Moscoso, M. (1996). Reacciones emocionales del estrés: ansiedad y cólera Avances en Psicología Clínica Latinoamericana.

Spielberger, Ch. y Reheiser, E. (1998). Medición del estrés en el trabajo: La encuesta de estrés laboral. Revista de Psicología. UNMSM. III(4).

Valdez, M. y De Flores, T. (1985). Psicobiología del estrés. Madrid: Martínez Roca.

Valdez, N. (1999). Estrés y Recursos de afrontamiento en grupo de adolescentes embarazadas. Lima: PUCP. 